\title{
DNA vaccine VGX-3100 with electroporation induces regression of cervical intraepithelial neoplasia 2/3 and clears HPV infection with robust $T$ cell responses: results of a randomized, double-blind, placebo-controlled Phase II trial
}

\author{
Laurent Humeau ${ }^{1 *}$, Cornelia Trimble², Matthew Morrow ${ }^{1}$, Xuefei Shen ${ }^{1}$, Michael Dallas', David Weiner ${ }^{3}$, \\ Jean Boyer ${ }^{3}$, Jian Yan ${ }^{1}$, Kimberly Kraynyak ${ }^{1}$, Albert Sylvester ${ }^{1}$, Mary Giffear ${ }^{1}$, Kathy Marcozzi-Pierce ${ }^{1}$, Divya Shah ${ }^{1}$, \\ Kate Broderick', Amir Khan', Jessica Lee ${ }^{1}$, Niranjan Sardesai', Mark Bargarazzi', the HPV-003 Protocol team ${ }^{1}$
}

From Society for Immunotherapy of Cancer 29th Annual Meeting

National Harbor, MD, USA. 6-9 November 2014

The Phase II study, designated HPV-003 (NCT01304524), assessed the safety and efficacy of VGX-3100 in 167 women with biopsy-proven cervical intraepithelial neoplasia (CIN) 2 or CIN 3 and HPV-16 or -18 . The randomized, placebo-controlled, double-blind study, was stratified by age and severity of CIN and evaluated cervical tissue changes after three $6 \mathrm{mg}$ intramuscular doses of the DNA vaccine VGX-3100 followed by electroporation (EP) with Inovio's CELLECTRA ${ }^{\circledR} 2000$ device at weeks 0 , 4, and 12. Cervical tissue was examined before starting blinded treatment and $\sim 9$ months later. The primary endpoint was regression of CIN 2 or CIN 3 to CIN 1 or no disease at 6 months post third dose. In the per-protocol population (PPP), lesions regressed in 53 of the 107 women receiving VGX-3100 (49.5\%) as compared to 11 of the 36 women in the placebo group $(\mathrm{p}<0.025)$. The secondary endpoint was to demonstrate HPV-16 or -18 clearance from the cervix in conjunction with regression of CIN 2/3 to CIN1 or no disease. Among the 107 women in the VGX-3100 group, 43 demonstrated regression and virological clearance (40.2\%), compared to 5 out of $35(14.3 \%)$ in the placebo group ( $\mathrm{p}<0.025)$.

The study also explored cell mediated immune responses to VGX-3100 in blood samples taken prior to the first vaccine dose and periodically thereafter. IFN- $\gamma$ ELISpot results revealed higher responses in the VGX-3100 treated group than in the placebo group. Flow cytometry and immunohistochemistry analyses are also ongoing. Finally, subjects were also monitored for tolerability and safety. The treatment was generally well-tolerated, with only administration site redness occurring significantly more frequently in the VGX-3100 group compared to the placebo group in the 7- and 28-day periods following treatment.

Altogether, the successful Phase II results clearly illustrate the highly promising potential of therapeutic vaccination with DNA followed by electroporation for the treatment of HPV-related precancerous cervical disease in women as well as HPV-associated cervical, head and neck, and anogenital cancers.

\section{Authors' details}

${ }^{1}$ Inovio Pharmaceuticals, USA. ${ }^{2}$ Johns Hopkins, Baltimore MD, USA. ${ }^{3}$ Perelman School of Medicine University of Pennsylvania, PA, USA.

Published: 6 November 2014

doi:10.1186/2051-1426-2-S3-017

Cite this article as: Humeau et al:: DNA vaccine VGX-3100 with electroporation induces regression of cervical intraepithelial neoplasia $2 / 3$ and clears HPV infection with robust T cell responses: results of a randomized, double-blind, placebo-controlled Phase II trial. Journal for ImmunoTherapy of Cancer 2014 2(Suppl 3):017.

Inovio Pharmaceuticals, USA

Full list of author information is available at the end of the article 